ARTICLE

https://doi.org/10.1038/s41467-019-10939-x

\title{
Angular momentum transfer from photon polarization to an electron spin in a gate-defined quantum dot
}

Takafumi Fujita1,2, Kazuhiro Morimoto1, Haruki Kiyama1,2, Giles Allison (1) 1,3, Marcus Larsson', Arne Ludwig (D) 4 , Sascha R. Valentin ${ }^{4}$, Andreas D. Wieck (10 ${ }^{4}$, Akira Oiwa ${ }^{2,5,6}$ \& Seigo Tarucha ${ }^{1,3}$

Gate-defined quantum dots (QDs) are such a highly-tunable quantum system in which single spins can be electrically coupled, manipulated, and measured. However, the spins in gatedefined QDs are lacking its interface to free-space photons. Here, we verify that a circularlypolarized single photon can excite a single electron spin via the transfer of angular momentum, measured using Pauli spin blockade (PSB) in a double QD. We monitor the interdot charge tunneling which only occur when the photo-electron spin in one QD is anti-parallel to the electron spin in the other. This allows us to detect single photo-electrons in the spinup/down basis using PSB. The photon polarization dependence of the excited spin state was finally confirmed for the heavy-hole exciton excitation. The angular momentum transfer observed here is a fundamental step providing a route to instant injection of spins, distributing single spin information, and possibly towards extending quantum communication.

\footnotetext{
${ }^{1}$ Department of Applied Physics, The University of Tokyo, 7-3-1 Hongo, Bunkyo-ku, Tokyo 113-8656, Japan. ${ }^{2}$ The Institute of Scientific and Industrial Research, Osaka University, 8-1 Mihogaoka, Ibaraki, Osaka 567-0047, Japan. ${ }^{3}$ Center for Emergent Matter Science (CEMS), RIKEN, 2-1 Hirosawa, Wako-shi, Saitama 351-0198, Japan. ${ }^{4}$ Lehrstuhl für Angewandte Festkörperphysik, Ruhr-Universität Bochum, Universitätsstraße 150, Gebäude NB, D-44780 Bochum, Germany. ${ }^{5}$ Center for Spintronics Research Network (CSRN), Graduate School of Engineering Science, Osaka University, Osaka 565-0871, Japan. ${ }^{6}$ Quantum Information and Quantum Biology Division, Institute for Open and Transdisciplinary Research Initiatives, Osaka University, Osaka 565-0871, Japan. Correspondence and requests for materials should be addressed to T.F. (email: fujita@sanken.oaska-u.ac.jp)
} 
S emiconductor quantum dots (QDs) is an appropriate platform for hosting photons and spins and therefore can provide a quantum interface for converting information media between photon polarization and spin orientation ${ }^{1-9}$. This process follows the selection rule in optical transitions to create an electron-hole pair or an exciton from a photon polarization while preserving angular momenta, and has long been studied for quantum wells by optical means ${ }^{10-12}$. However, to assess a photon-to-spin interface, it is necessary to create the single electron spins with various photon polarization and electrically measure the spin orientation before it relaxes. Laterally gated QDs in a quantum well may be appropriate for these purposes because the orbital and spin angular momenta are well-defined by the strong vertical confinement and suitable for the transition with angular momentum selection rule, while the soft lateral confinement will least affect the selection rule for the optical excitation. Investigating the singly excited charged spin electrically is essential for understanding the selection rule in the optical excitation, robustness of the photo-generated electron spin, and for applications in nano-scaled spin-based information processing ${ }^{13,14}$, but a challenging task.

Single electrons are optically accessible in gate-defined QDs because the electronic potential acts as a trapping potential for the electrons, while it is repulsive for the holes upon single electron-hole pair excitation in the QD area ${ }^{15,16}$. Then single photo-electrons can be generated from the individual heavy-hole $(\mathrm{HH})$ and light-hole $(\mathrm{LH})$ excitons ${ }^{17,18}$, and detected using a nearby charge sensor because the photo-electron trapping results in a charge addition for the dot. The photo-electron spin orientation can be judged using the spin-dependent tunneling from the dot to the lead whose Fermi energy is aligned between the Zeeman sub-levels in the $\operatorname{dot}^{19}$. However, the Fermi energy has been known to fluctuate due to the photon absorption in the leads outside the dot which has hindered the detection of the spin state ${ }^{15-17}$

In this work, we measure the single excited spin of a photoelectron in real-time using Pauli spin blockade (PSB) spin readout. We adjust a double QD (DQD) to the inter-dot tunneling resonance condition, which is sensitive to the energy misalignment between the dots. The photo-excited charge moving backand-forth between the dots enables us to keep track of the suitable condition for a PSB spin readout even when the Fermi energies of the leads connected to either the DQD or charge sensor fluctuate. Finally using this reliable condition, we verify the principle selection rule from a single circularly polarized photon to the excited single electron spin state at the $\mathrm{HH}$ exciton by rotating the incident polarization.

\section{Results}

Optical selection rules of GaAs HH states. Figure 1a depicts the conversion rule that holds for the QW device. We here focus on the conservation of angular momentum in the electron-hole pair photo-excitation. We photo-excite the lowest energy $\mathrm{HH}$ state for simplicity and also to achieve the high efficiency of excitation. The numbers in the figure represent the angular momenta that are defined as positive (negative) for parallel (anti-parallel) to the light propagation direction. The angular momentum is conserved in the optical transition such that a photon with angular momentum -1 $(+1)$ for $\sigma^{-}\left(\sigma^{+}\right)$polarization, creates an electron with spin $+1 / 2$ $(-1 / 2)$ and a $\mathrm{HH}$ with projection of total angular momentum $-3 / 2$ $(+3 / 2)$, all expressed in units of $\hbar$. In other words, $\mid \sigma^{-}>_{\mathrm{ph}} \rightarrow$ $\left|+1 / 2>_{\mathrm{e}} \otimes\right|-3 / 2>_{\mathrm{HH}},\left|\sigma^{+}>_{\mathrm{ph}} \rightarrow\right|-1 / 2>_{\mathrm{e}} \otimes \mid+3 / 2>_{\mathrm{HH}}$.

Single-spin detection using PSB. For measuring the photogenerated single electron spin, we utilize the mechanism of PSB a
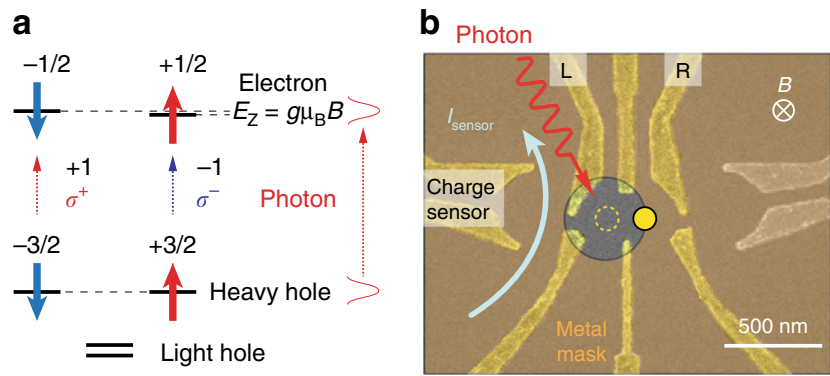

C

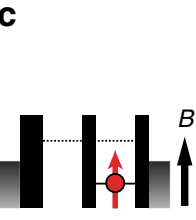

$(0,1)$

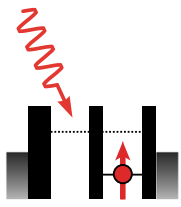

$(0,1) \rightarrow(1,1)$

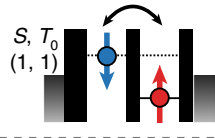

Spin down

$(1,1) \leftrightarrow(0,2)$

(i) Initialization

(ii) Irradiation

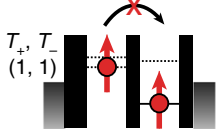

Spin up

$(1,1)$

Fig. 1 Photo-electron spin detection scheme. a Angular momentum conservation in the heavy-hole band excitation. The numbers are the angular momenta of the respective particles in units of $\hbar$. $\mathbf{b}$ Scanningelectron-micrograph picture of the double quantum dot (DQD) gate pattern nominally identical to the measured sample. We mainly use the charge sensor quantum dot (QD) formed on the left. The actual sample has a metal mask placed on an insulator film. Photons are irradiated onto the left dot through an aperture in the metal mask in transparent yellow. c Sequence of the single photo-electron spin measurement: (i) Initialization to $(0, \uparrow)$ by waiting long enough at the $(0,1)$ charge state, (ii) exciting an electron-hole pair. We post-select the events where a single electron is captured in the left QD, and (iii) single photo-electron-spin measurement. Spin-down and up are distinguished by observing the charge change due to the $(1,1)-(0,2)$ transition. The DQD is initialized back to $(0, \uparrow)$ by waiting for the excess electron to escape from the DQD

while monitoring the electron move inside the DQD (Fig. 1b, c). First we place a single electron on the right dot and then the electron spin is initialized to the up-spin state under a sufficiently large magnetic field. This sets the read-out basis vectors of up/ down spin for the photo-electron. The left dot is emptied for the photo-electron to be trapped. After a successful trapping, the DQD forms $(1,1)$, where $(n, m)$ denotes the set of electron numbers in the left and right QD, respectively. The $(1,1)$ and $(0,2)$ state simultaneously align upon the single photo-electron trapping, energetically allowing transition between the two states ${ }^{20}$. This transition is repeated when the two spins are anti-parallel, but blocked when they are parallel because of the PSB mechanism (explained below). Considering again the readout basis, this difference allows us to measure the spin orientation of the photoelectron as up or down, respectively. Note that the hole is also photo-created in the dot but quickly escapes to the reservoir in the QW ${ }^{16}$

PSB prohibits charge tunneling between the dots when the tunneling requires spin flip. Starting from the tunnel-coupled two-electron state of $(1,1)$, the ground state is either a spin-singlet state, $S=(|\uparrow>| \downarrow>-|\downarrow>| \uparrow>) / \sqrt{2}$, or a spin-triplet state of $T_{0}=$ $\left.(|\uparrow>| \downarrow\rangle+|\downarrow>| \uparrow>) / \sqrt{2}, \quad T_{+}=|\uparrow>| \uparrow\right\rangle$, and $T_{-}=|\downarrow\rangle|\downarrow\rangle$. Due to PSB, only $S(1,1)$ can make a transition to the $(0,2)$ state that is $S$ $(0,2)$. In the absence of an external magnetic field all these spin states can be mixed up because of the fluctuating Overhauser field by a few $\mathrm{mT}$ in the abundant nuclear spin bath ${ }^{21}$. Therefore, for any spin states of $(1,1)$, the electron inter-dot tunneling time is more or less the same. In the presence of an external magnetic 
a

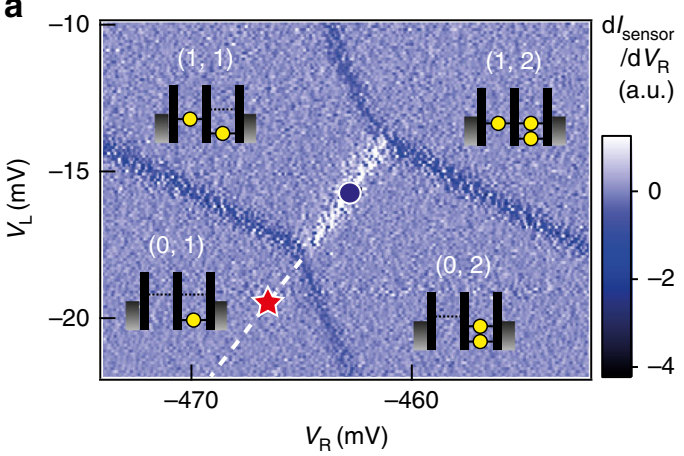

d

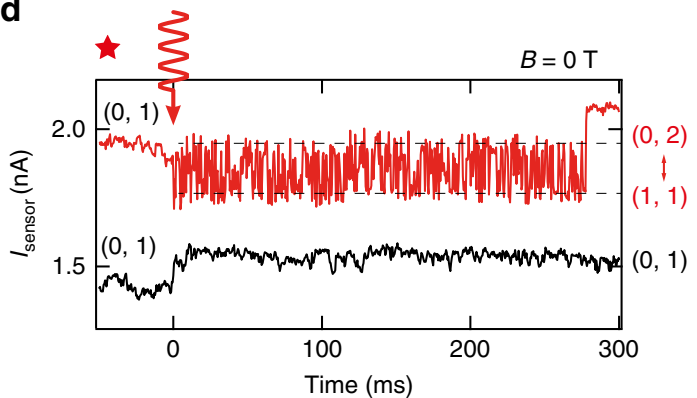

b

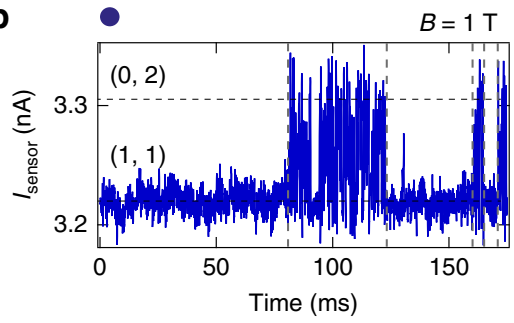

C

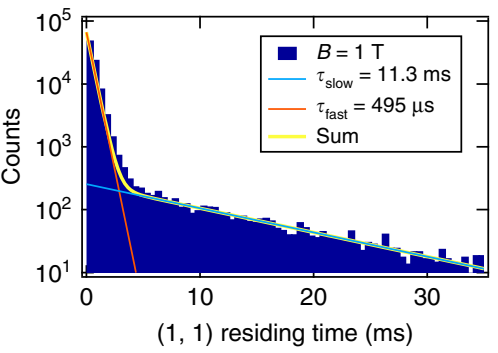

e

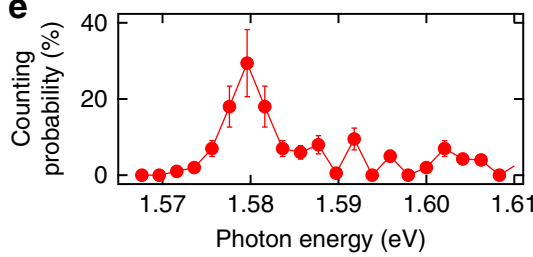

Fig. 2 Real-time detection of Pauli spin blockade and single-shot photo-electron trapping. a Charge stability diagram measured around the $(1,1)$ - $(0,2)$ transition line in white and the $(0,1)$ region. $\mathbf{b}$ Example trace of a real-time charge sensor current $I_{\text {sensor }}$ measured at point $\mathbf{0}$ in the stability diagram with $B=1 \mathrm{~T}$. The trace starts from the $I_{\text {sensor }}$ blocked at a lower value indicating $(1,1)$ and frequently changes between the low and high values indicating the repeated $(1,1)-(0,2)$ transitions. The two regions indicate the parallel and anti-parallel spin configurations, respectively. $\mathbf{c}$ Histogram of the number of events of finding the $(1,1)$ blocked state vs. the state residing time derived from the $I_{\text {sensor }}$ trace measured continuously for minutes. The histogram fits a double-exponential curve with two time constants, $\tau_{\text {slow }}$ and $\tau_{\text {fast. }}$. These values are used to optimize threshold time for the single-shot spin measurements (see text). d Photon irradiation results at $B=0$ T measured at point $\star$ in the stability diagram. $I_{\text {sensor }}$ in red oscillates due to the repeated $(1,1)$ - $(0,2)$ transitions. The two-electron charge dynamics is observed until one of the two electrons escapes from the DQD. $I_{\text {sensor }}$ in black stays at the low level showing no photo-electron trapping. A small offset of $I_{\text {sensor }}$ observed for $t>0 \mathrm{~ms}$ is due to the small photoconductivity of the charge sensor. e Energy spectrum of the photo-electron trapping probability. The laser power is tuned such that 20 photons reach the QD area per shot for this figure. The heavyhole peak is found at $1.579 \mathrm{eV}$, and the light hole peak is expected to be at $1.602 \mathrm{eV}$ from simulation for the $7.3 \mathrm{~nm}$-width quantum well (QW) but not well resolved. Error bars are standard deviations expected from the binomially distributed single-shot results of photo-electron detection

field well exceeding a few $\mathrm{mT}$, the $T_{+}$and $T_{-}$states are separated by Zeeman energy, while the $S$ and $T_{0}$ states of $(1,1)$ both having the zero magnetic moment are still degenerate. In such a condition the inter-dot electron tunneling is spin blocked for the parallel spin-triplet states, whereas not for the non-magnetic antiparallel spin states. This difference can be distinguished by charge sensing within the spin relaxation time.

We carefully adjust the gate voltages of the DQD device to make appropriate the dot potentials, dot-lead and dot-dot tunnel couplings, and Zeeman energies for the spin readout measurement ${ }^{19,22}$. Then the measured charge stability diagram in the vicinity of the inter-dot transition line between $(1,1)$ and $(0,2)$ is shown in Fig. 2a. We set the gate bias to point on the border of $(1,1)$ and $(0,2)$ to monitor the electron inter-dot tunneling events. Here we set the inter-dot tunneling times above milliseconds longer than the integration time of the charge sensor to observe the inter-dot electron move in real time. Figure $2 \mathrm{~b}$ shows an example of the time-dependent charge sensor current $I_{\text {sensor }}$ for $B=1 \mathrm{~T}$. We observe two conditional regions: the first shows a number of consecutive $I_{\text {sensor }}$ spikes indicating repeated transitions between the spin-anti-parallel states of $(1,1)$ and $(0,2)$, while the second shows $I_{\text {sensor }}$ staying at the low-level indicating the spin-parallel $(1,1)$ states blocked due to PSB. We measure the $(1,1)$ residing time and count the number of events to construct a histogram of the $(1,1)$ residing time. We can fit the histogram to a double-exponential curve with two time constants (Fig. 2c): blockade lifetime $\tau_{\text {slow }}$ of parallel spins and spin-preserved tunneling time $\tau_{\text {fast }}$ of anti-parallel spins. We use the ratio of $\tau_{\text {slow }}$ and $\tau_{\text {fast }}$ to calculate a suitable threshold time for judging the spin orientation from the charge sensing data so as to minimize the spin measurement error for arbitrary two-electron states of $(1,1)$ (see Supplementary Note 2 for details). In addition, the read-out fidelity can be increased by making the magnetic field larger than that arising from the fluctuating hyperfine interaction and spin-orbit interaction ${ }^{19,22}$.

Single photo-electron trapping and its spin detection. In contrast, when trapping a photo-electron, an electron is added to the dot whose chemical potential is located above the Fermi level of the reservoir. Such a non-equilibrium state does not appear in the stability diagram in Fig. 2a, but it is still possible to approximate the gate voltages needed to make alignment of $(1,1)$ and $(0,2)$ upon photo-excitation, for instance those at point $\star$, which is inside the $(0,1)$ region and along the dashed line. Figure $2 \mathrm{~d}$ shows typical $I_{\text {sensor }}$ responses when a laser pulse is irradiated at $t=0$ with $B=0 \mathrm{~T}$, showing a suitable single photo-electron trapping in the upper trace and no photo-electron trapping in the lower trace. We reconfirm the $(1,1)-(0,2)$ degenerate condition by observing the back-and-forth inter-dot electron tunneling events 
subsequent to the photon irradiation. The escape time of the excess electron is about a few $100 \mathrm{~ms}$ restricted by the thicker outer barrier. We note that after retaining the initial $(0,1)$ state no electron enters the DQD from the reservoir, assuring the charge change observed in $I_{\text {sensor }}$ is only due the photo-electron trapping. The small feature in $I_{\text {sensor }}$ upon irradiation is attributed to the effect of persistent photo-conductivity, where we see an increase step of $I_{\text {sensor }}$ from the low-level followed by a gradual decrease. The persistent component accumulates in every shot but is partially compensated by weakly illuminating a longer wavelength light (here a $1550 \mathrm{~nm}$ wavelength light from a light-emitting diode) to de-excite the impurities in the heterostructure ${ }^{23}$. Additionally, trapping of multiple photo-electrons can also be detected with a very small probability, and therefore we disregard such events ${ }^{20}$. Figure $2 \mathrm{e}$ shows the counting probability of detecting the photo-electron trapping events as a function of the incident photon energy. The spectrum indicates the peak position of the $\mathrm{HH}$ exciton at $1.579 \mathrm{eV}$. We set the photon energy to this peak in the experiment of spin readout of a single photo-electron. The $\mathrm{LH}$ exciton is expected to be at around $1.602 \mathrm{eV}$ from simulation and additional measurements ${ }^{18}$, but not well resolved.

Angular momentum transfer. We finally perform the photoelectron trapping experiment to study the angular momentum transfer from a circularly polarized photon to an electron spin. The magnetic field $B$ is increased to $1.65 \mathrm{~T}$ to facilitate the initialization of $(0,1)$ having a spin-up electron (i.e. $(0, \uparrow))$ by setting Zeeman energy larger than the electron temperature. Figure $3 a$ shows the anti-parallel (parallel) spin state detection upon irradiating linearly polarized photons, in the upper (lower) panel of $I_{\text {sensor. }}$ The photo-generation of the $(1,1)$ anti-parallel spin state is discerned by observing the repeated events of inter-dot electron tunneling while keeping the spin orientation, and then relaxing to the $(1,1)$ parallel spin state as a spin-blocked state, and finally restoring the initial $(0,1)$ state by the escape of one electron to the lead. In contrast, the lower panel of $I_{\text {sensor }}$ shows the opposite behavior, indicating the photo-generation of the parallel spin state $T_{+}$. Given that $(0, \uparrow)$ is initially formed, by the photo-

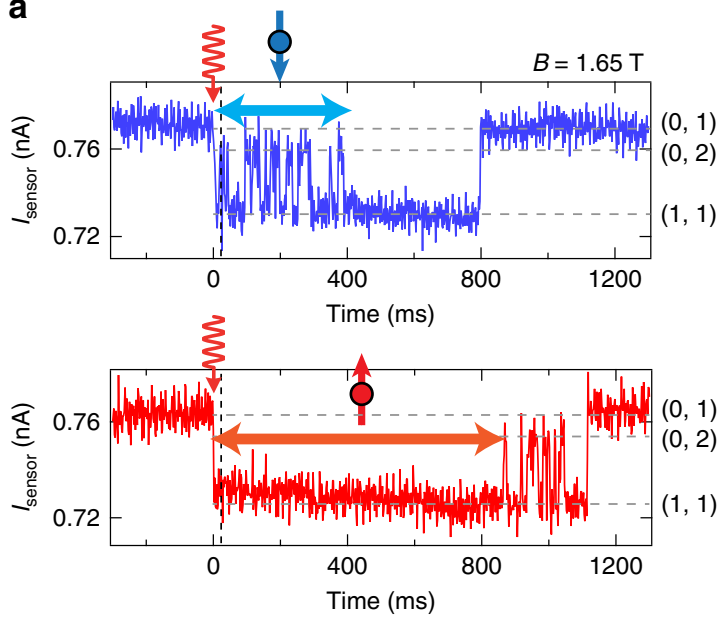

excitation the upper (lower) panel indicates the photo-generation of a spin-down (spin-up) electron. $B$ in a perpendicular configuration cannot be very large, otherwise the orbital excited $(0,2)$ spin-triplet states become so close to $S(0,2)$ in energy that the blocked $(1,1)$ triplet state is relaxed to the $(0,2)$ triplet state (see Supplementary Note 3 and Supplementary Fig. 2 for additional examples of photo-electron blockade signals in the lower intermediate fields).

Figure $3 \mathrm{~b}$ shows the main result on the probability of detecting a photo-electron blockade signal in $I_{\text {sensor }}$ (blocked probability) for five different polarizations and two opposite magnetic fields. Each data point is obtained from $\sim 50$ photo-electron trapping signals out of $\sim 1000$ or more pulse irradiations. We set a threshold time of $\sim 10 \mathrm{~ms}$ to distinguish the PSB signal from the signal of the antiparallel spin transition of $(1,1)-(0,2)$. The obtained PSB probabilities show a sinusoidal dependence when changing the photon polarization from the left-handed to the right-handed circular polarization, $\sigma^{-}$to $\sigma^{+}$via $H$ linear polarization. Additionally, the probability curve shows an opposite behavior when the polarity of the magnetic field is reversed. This can happen because the initialized spin becomes opposite, while the photo-generated electron spin is not affected. The correspondence between polarization and spin indicates that a $\sigma^{-}\left(\sigma^{+}\right)$photon creates an electron spin pointing in the positive (negative) direction, which is expected from the angular momentum transfer in the $\mathrm{HH}$ band excitation depicted in Fig. 1a.

\section{Discussion}

Here we discuss the fidelity of the angular momentum transfer. We evaluate the fidelity of $63 \%$ by comparing the ideal detection against the worst case of measuring the opposite spin in Fig. $3 \mathrm{~b}$. The probabilities used for the calculation are averaged over the four combinations of circular polarization and field, e.g. $\sigma^{-}$and $\sigma^{+}$polarization and positive and negative fields for detecting the spin-blocked signal, and the same for detecting the un-blocked signal. The two most likely errors come from spin-relaxation during the measurement stage and thermalization in the $(0, \uparrow)$ initialization stage. From measurement of the tunneling time

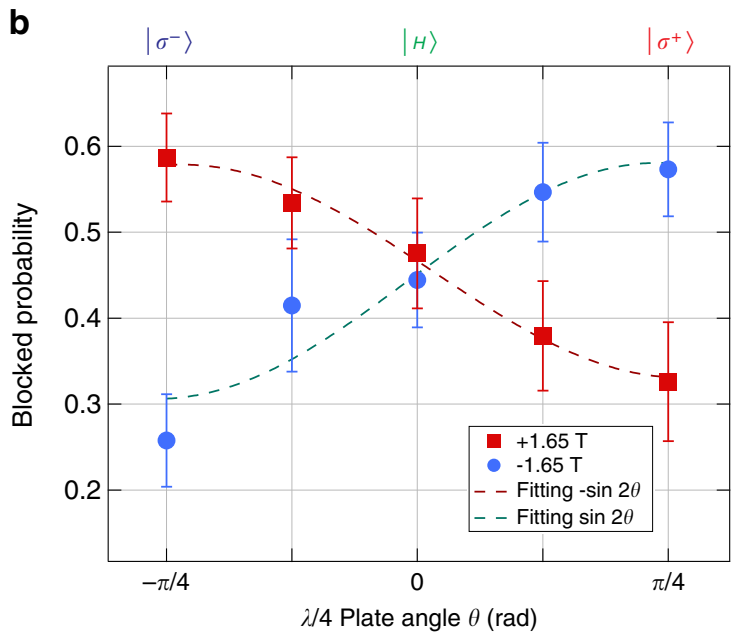

Fig. 3 Single photo-electron spin detection and angular momentum transfer. a Two typical $I_{\text {sensor }}$ traces of photo-electron trapping, indicating down-spin trapping in the upper panel, and up-spin trapping in the lower panel, respectively. $\mathbf{b}$ Polarization and field polarity dependence of the blocked probability, reflecting the angular momentum transfer from a single photon to an electron spin. A sinusoidal dependence is observed against the rotation angle $\theta$ of polarization from $-\pi / 4$ to $\pi / 4$. By reversing the external magnetic field or reversing the polarity of the spin detection axis, we observe a flipped curve. This means that a single photon polarization creates a single fixed electron spin in the dot, following the conversion rule depicted in Fig. 1a. Each data point is derived from the single-shot measurements repeated more than 1000 times. The photo-electron trapping rate averaged over all measurements is $3 \%$. The error bars $~ 5 \%$ are the standard deviation of the binomial distribution for each measurement point. From the fitting to the sinusoidal curve an amplitude of $25 \pm 2 \%(27 \pm 5 \%)$ and an offset of $45 \pm 1 \%(44 \pm 2 \%)$ for $B=1.65 \mathrm{~T}(-1.65 \mathrm{~T})$ are obtained. We used these parameters for fidelity calculation 
constants with no irradiation, we obtain $\tau_{\text {slow }}=324 \pm 1 \mathrm{~ms}$ (corresponding to the parallel spin $T_{1}$ ) and $\tau_{\text {fast }}=5.68 \pm 0.02 \mathrm{~ms}$, and calculate each fidelity of $93.0 \%(98.4 \%)$ for the parallel (antiparallel) spin detection. Here we ignore the signal integration time. Asymmetry of the fidelity between the two spin configurations appears when we minimize the average error by adjusting the threshold time (see Supplementary Note 2 for derivations). Next for the thermal initialization, we prepare the $(0, \uparrow)$ state just by keeping an electron in the right dot, in a similar manner to previous work performed using an optical technique ${ }^{24,25}$. From the electron temperature of $100 \mathrm{mK}$ (8.6 $\mu \mathrm{eV}$ ) and Zeeman energy of $11.3 \mu \mathrm{eV}$ in our experiment we evaluate the initialization fidelity of $79 \%$. This error may be reduced by increasing the magnetic field of the in-plane direction but not the out-of-plane direction. We may further improve the fidelity, measurement time, and robustness of spin detection by utilizing this spin non-destructiveness 26,27 .

For the $\mathrm{HH}$ excitation in this condition, we think of the angular momentum transfer as a classical condition. The $\mathrm{HH}$ spin splitting is negligible due to zero $g$-factor, therefore if we irradiate a superposition of $\left|\sigma^{-}\right\rangle_{\mathrm{ph}}$ and $\left|\sigma^{+}\right\rangle_{\mathrm{ph}}$, this state has access to both spins of the $\mathrm{HH}$ and electrons ${ }^{13}$. The resulting electron spin will be entangled with the hole spin upon excitation, i.e. $\alpha\left|\sigma^{-}>_{\mathrm{ph}}+\beta\right| \sigma^{+}>_{\mathrm{ph}} \rightarrow$ $\alpha\left|+1 / 2>_{\mathrm{e}} \otimes\right|-3 / 2>_{\mathrm{HH}}+\beta\left|-1 / 2>_{\mathrm{e}} \otimes\right|+3 / 2>_{\mathrm{HH}}$. Then due to the short coherence time of the hole spin, the electron spin would also lose its phase information without interacting with its environment ${ }^{28}$. Meanwhile, the electron spin orientation may well be preserved because the $\mathrm{HH}$ escapes to the environment in a short time such that the spin exchange with the electron spin is small. Our observation in the PSB probability peaking at the circular polarization supports this claim of electron spin preservation. In the future, the transfer to spin orientation could be combined with down converted pair of photons, that can form a pair of single photon and a photo-electron pair ${ }^{29}$, for generation of classically correlated single spins. Another important path is to preserve the electron spin phase information after hole separation ${ }^{28}$. The requirement is to excite one of the spin-split $\mathrm{LH}$ band under an inplane magnetic field to disentangle the hole spins while achieving symmetric excitation of the spin basis. After such feasible adjustment, the transfer can be applied for measuring entangled spin pairs and for efficient long-distant quantum communication using gatedefined QDs ${ }^{13,30,31}$.

In conclusion, we observed that the single electron spin created from a single circularly polarized photon has a correlation expected from the selection rules deduced from the quantum well structure of the host GaAs. Utilizing the two-electron spin blockade in the tunnel resonant condition served to project a single spin created by the photon and to justify the stability of the energy levels during detection. This progress may be integrated with the fast and remote optical investigations of single spins, which is promising as a classical and quantum nano-scale electronic platform.

\section{Methods}

Measurement sample. A DQD and a nearby QD charge sensor are fabricated in the QW. The QDs are defined by the surface Schottky gate electrodes shown in Fig. 1b. The current through the charge sensor, $I_{\text {sensor }}$, detects the change in the charge configuration due to the event of photo-electron trapping in the DQD with a response time of $100 \mu$ s or less. Photon pulses with width of 3 ps are produced from a wavelength-tunable Ti:sapphire laser, and irradiated onto left dot through a 400 -nm-diameter aperture in the metal mask. The device is placed in an optically accessible dilution refrigerator with base temperature of $25 \mathrm{mK}$. The QW wafer used here has a 7.3-nm-thick GaAs QW layer between a $95 \mathrm{~nm}$-thick $\mathrm{Al}_{0.34} \mathrm{Ga}_{0.66} \mathrm{As}$ barrier layer above and a 100 -nm-thick $\mathrm{Al}_{0.34} \mathrm{Ga}_{0.66} \mathrm{As}$ barrier layer beneath. The bottom thick barrier avoids excess photo-electrons coming from a thick GaAs buffer beneath the QW. An external magnetic field $B$ is applied in the out-of-plane direction to the DQD device. The electron $g$-factor of the QW estimated for this field direction is $\left|g_{\perp}\right|=0.12^{32}$ (see Supplementary Note 1 and Supplementary Fig. 1 for more details about the electronics and optics).
}

\section{Data availability}

The authors declare that the data supporting the findings of this study are available within the paper and its supplementary information files.

Received: 27 March 2015 Accepted: 3 June 2019

Published online: 16 July 2019

\section{References}

1. Petta, J. R. et al. Coherent manipulation of coupled electron spins in semiconductor quantum dots. Science 309, 2180-2184 (2005).

2. Nowack, K. C., Koppens, F. H. L., Nazarov, Y. V. \& Vandersypen, L. M. K. Coherent control of a single electron spin with electric fields. Science 318, 1430-1433 (2007)

3. Brunner, R. Two-qubit gate of combined single-spin rotation and interdot spin exchange in a double quantum dot. Phys. Rev. Lett. 107, 146804 (2011)

4. Kawakami, E. et al. Electrical control of a long-lived spin qubit in a $\mathrm{Si} / \mathrm{SiGe}$ quantum dot. Nat. Nanotechnol. 9, 666-670 (2014).

5. Takeda, K. A fault-tolerant addressable spin qubit in a natural silicon quantum dot. Sci. Adv. 2, e1600694 (2016).

6. Yoneda, J. et al. A quantum-dot spin qubit with coherence limited by charge noise and fidelity higher than 99.9\%. Nat. Nanotechnol. 13, 102-106 (2018).

7. Shulman, M. D. Suppressing qubit dephasing using real-time Hamiltonian estimation. Nat. Commun. 5, 5156 (2014).

8. Noiri, A. Coherent electron-spin-resonance manipulation of three individual spins in a triple quantum dot. Appl. Phys. Lett. 108, 153101 (2016).

9. Otsuka, T. et al. Single-electron spin resonance in a quadruple quantum dot. Sci. Rep. 6, 31820 (2016).

10. Meier, F. \& Zakharchenya, B. P. (eds). Optical Orientation (Elsevier, Amsterdam, 1984).

11. Crooker, S. A., Awschalom, D. D., Baumberg, J. J., Flack, F. \& Samarth, N. Optical spin resonance and transverse spin relaxation in magnetic semiconductor quantum wells. Phys. Rev. B 56, 7574-7588 (1997).

12. Koralek, J. D. et al. Emergence of the persistent spin helix in semiconductor quantum wells. Nature 458, 610-613 (2009).

13. Vrijen, R. \& Yablonovitch, E. A spin-coherent semiconductor photo-detector for quantum communication. Physica E 10, 569-575 (2001).

14. Awschalom, D. D., Samarth, N. \& Loss, D. Semiconductor Spintronics and Quantum Computation (Springer, Berlin, Heidelberg, 2002).

15. Rao, D. S., Szkopek, T., Robinson, H. D., Yablonovitch, E. \& Jiang, H.-W. Single photoelectron trapping, storage, and detection in a one-electron quantum dot. J. Appl. Phys. 98, 114507 (2005).

16. Pioda, A. et al. Single-shot detection of electrons generated by individual photons in a tunable lateral quantum dot. Phys. Rev. Lett. 106, 146804 (2011).

17. Kuwahara, M., Kutsuwa, T., Ono, K. \& Kosaka, H. Single charge detection of an electron created by a photon in a g-factor engineered quantum dot. Appl. Phys. Lett. 96, 163107 (2010).

18. Morimoto, K. et al. Single photoelectron detection after selective excitation of electron heavy-hole and electron light-hole pairs in double quantum dots. Phys. Rev. B 90, 085306 (2014).

19. Maisi, V. F. et al. Spin-orbit coupling at the level of a single electron. Phys. Rev. Lett. 116, 136803 (2016).

20. Fujita, T. et al. Nondestructive real-time measurement of charge and spin dynamics of photoelectrons in a double quantum dot. Phys. Rev. Lett. 110, 266803 (2013)

21. Merkulov, I. A., Efros, A. L. \& Rosen, M. Electron spin relaxation by nuclei in semiconductor quantum dots. Phys. Rev. B 65, 205309 (2002).

22. Fujita, T. et al. Signatures of hyperfine, spin-orbit, and decoherence effects in a Pauli spin blockade. Phys. Rev. Lett. 117, 206802 (2016).

23. He, L. X., Martin, K. P. \& Higgins, R. J. Infrared quenching of persistent photoconductivity in $\mathrm{GaAs} / \mathrm{Al}_{\mathrm{x}} \mathrm{Ga}_{1-\mathrm{x}} \mathrm{As}$ heterostructures. Phys. Rev. B 39, 1808-1818 (1989).

24. Högele, A. et al. Spin-selective optical absorption of singly charged excitons in a quantum dot. Appl. Phys. Lett. 86, 221905 (2005).

25. Kroutvar, M. et al. Optically programmable electron spin memory using semiconductor quantum dots. Nature 432, 81-84 (2004).

26. Pazy, E., Calarco, T. \& Zoller, P. Spin state readout by quantum jump technique: for the purpose of quantum computing. IEEE Trans. Nanotechnol. 3, 10-16 (2004)

27. Meunier, T. et al. Nondestructive measurement of electron spins in a quantum dot. Phys. Rev. B 74, 195303 (2006).

28. Kosaka, H. et al. Coherent transfer of light polarization to electron spins in a semiconductor. Phys. Rev. Lett. 100, 096602 (2008).

29. Kuroyama, K. et al. Single electron-photon pair creation from a single polarization-entangled photon pairSci. Rep. 7, 16968 (2017).

30. Briegel, H.-J., Dür, W., Cirac, J. \& Zoller, P. Quantum repeaters: the role of imperfect local operations in quantum communication. Phys. Rev. Lett. 81, 5932-5935 (1998). 
31. Gaudreau, L. et al. Entanglement distribution schemes employing coherent photon-to-spin conversion in semiconductor quantum dot circuits. Semicond. Sci. Technol. 32, 093001 (2017)

32. Allison, G. et al. Tuning the electrically evaluated electron Landé $g$ factor in GaAs quantum dots and quantum wells of different well widths. Phys. Rev. B 90, 235310 (2014).

\section{Acknowledgements}

We acknowledge T. Asayama for his contribution to the initial stage of this work. This work was supported by Grants-in-Aid for Scientific Research S (26220710 and 17H06120), Innovative Areas "Nano Spin Conversion Science" (Grant No. 26103004), ImPACT Program of Council for Science, Technology and Innovation (Cabinet Office, Government of Japan), MEXT Project for Developing Innovation Systems, and QPEC, The University of Tokyo, JST CREST (JPMJCR15N2, JPMJCR1675), Dynamic Alliance for Open Innovation Bridging Human, Environment and Materials. T.F. and H.K. were supported by JSPS Research Fellowships for Young Scientists. T.F. was supported by Yukawa Memorial Foundation (Mochizuki Fund). M.L. acknowledges support as an "International Research Fellow of the Japan Society for the Promotion of Science". A.L. and A.D.W. acknowledge gratefully support from DFG-TRR 160, Mercur Pr-2013-0001, BMBF-Q.com-H 16KIS0109, and DFH/UFA CDFA-05-06.

\section{Author contributions}

T.F. performed the experiments, analyzed, and wrote the manuscript, K.M. fabricated the device, K.M., H.K., G.A. and M.L. confirmed the experimental foundation, A.L., S.R.V. and A.D.W. grew the wafer, A.O. and S.T. initiated and supervised the experimental work. All authors discussed the results and implications and commented on the manuscript at all stages.

\section{Additional information}

Supplementary Information accompanies this paper at https://doi.org/10.1038/s41467019-10939-x.

Competing interests: The authors declare no competing interests.

Reprints and permission information is available online at http://npg.nature.com/ reprintsandpermissions/

Peer review information: Nature Communications thanks the anonymous reviwers for their contribution to the peer review of this work.

Publisher's note: Springer Nature remains neutral with regard to jurisdictional claims in published maps and institutional affiliations.

(c) (i) Open Access This article is licensed under a Creative Commons Attribution 4.0 International License, which permits use, sharing, adaptation, distribution and reproduction in any medium or format, as long as you give appropriate credit to the original author(s) and the source, provide a link to the Creative Commons license, and indicate if changes were made. The images or other third party material in this article are included in the article's Creative Commons license, unless indicated otherwise in a credit line to the material. If material is not included in the article's Creative Commons license and your intended use is not permitted by statutory regulation or exceeds the permitted use, you will need to obtain permission directly from the copyright holder. To view a copy of this license, visit http://creativecommons.org/ licenses/by/4.0/.

(C) The Author(s) 2019 